\title{
A Novel Microwave Treatment to Augment the Mechanical Properties of Polymeric Materials ${ }^{\dagger}$
}

\author{
Swamini Chopra ${ }^{1}$, Kavita A. Deshmukh ${ }^{2}$, Kanchan Zine ${ }^{3}$, Kartik Rathod ${ }^{3}$, Abhay Deshmukh ${ }^{4}$, Dilip \\ Peshwe $^{2}$ \\ 1 Maharashtra Institute of Technology, Aurangabad, India \\ Visvesvaraya National Institute of Technology, Nagpur, India \\ College of Engineering, Pune, India \\ Rashtrasant Tukdoji Maharaj Nagpur University, Nagpur, India \\ * Correspondence: swamini.chopra@mit.asia; \\ $\dagger$ Presented at Materials Chemistry and Physics (Materials Chemistry 2020) - International e-Conference
}

Received: 16.09.2020; Revised: 20.09.2020; Accepted: 24.09.2020; Published: 27.09.2020

\begin{abstract}
The present study portrays a novel post-processing treatment by using microwave radiations for enhancing the mechanical properties of five commonly used engineering polymers, Poly-amide (PA), Poly-butylene-terephthalate (PBT), Poly-propylene (PP), Poly-carbonate (PC), Acrylonitrilebutadiene-styrene (ABS). The analysis revealed that the crystal structures of the polymers improved after the treatment due to a more favorable rearrangement of crystalline segments within the polymers. Furthermore, tensile properties and tribological performance of microwave treated polymers were found to be significantly better when compared to those of untreated counterparts. The tensile strength, elongation, and wear performance of PA increased by 51\%, 286\%, and 45\%, respectively, only after a treatment of 20 seconds. A similar response was also exhibited by other polymers as well. It was noted that the optimum time for microwave treatment could vary depending on the different crystalline nature of the polymers. The degree of randomness in the molecular chains of semi-crystalline polymers is less; thus, it requires less treatment time. However, for amorphous polymers, as randomness increases, more time is needed. As such, post-processing microwave treatment of polymers has proven beneficial as a cost-effective, time-saving, and environment-friendly technique for enhancing material properties significantly.
\end{abstract}

Keywords: post-processing treatment; microwave; polymers; structural rearrangement; tensile properties; tribological performance.

(C) 2020 by the authors. This article is an open-access article distributed under the terms and conditions of the Creative Commons Attribution (CC BY) license (https://creativecommons.org/licenses/by/4.0/).

\section{Funding}

This research received no external funding.

\section{Acknowledgments}

This research has no acknowledgment.

\section{Conflicts of Interest}

The authors declare no conflict of interest. 